\title{
The pyrrole approach towards the synthesis of fully functionalized cup-shaped molecules
}

\author{
Cristiano Zonta, ${ }^{*}$ Fabrizio Fabris and Ottorino De Lucchi \\ Dipartimento di Chimica, Università Ca' Foscari di Venezia
}

Dorsoduro 2137, I-30123 Venezia, Italy

zontac@unive.it

\section{Experimentals}

General. Reactions were carried out using standard techniques in flame-dried glassware cooled under argon. Commercial high purity reagents were employed without further purification. Dry THF was distilled prior use from sodium and benzophenone. Dry NMP was distilled prior to use from calcium hydride. Copper(I) 2-thiophenecarboxylate (CuTC) was obtained according to the procedure reported by Liebeskind. ${ }^{1}$ The progress of the reactions was monitored by TLC, GC-MS or ${ }^{1} \mathrm{H}$ NMR spectroscopy. Gas-chromatographic analyses were performed with a $30 \mathrm{~m}, 0.25 \mathrm{~mm}$ ID, Rtx-5MS capillary column (5:95 diphenyl-/dimethyl-polysiloxane). Flash-chromatographic purifications were performed with 230-400 mesh silica-gel Merk 60. Melting points are uncorrected. ${ }^{1} \mathrm{H}$ and ${ }^{13} \mathrm{C}$ NMR spectra were recorded on Bruker Avance-300 and Varian Unity 400 spectrometers. All the structures were determined by NMR studies through monodimensional experiments ${ }^{1} \mathrm{H}$ and ${ }^{13} \mathrm{C}$ NMR, and bidimensional experiment (COSY, NOESY, HMQC and HMBC). Microanalyses were performed on a Perkin Elmer 2400 CHN Elemental Analyser.

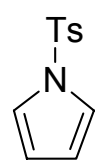

2

1-(Toluene-4-sulfonyl)-1H-pyrrole (2). Pyrrole (9.7 g, 0.149 mol) was added to a well-agitated suspension of $\mathrm{NaOH}(18.0 \mathrm{~g}, 0.596 \mathrm{~mol})$ in $100 \mathrm{~mL}$ of dichloroethane. This mixture was then cooled to $0{ }^{\circ} \mathrm{C}$ and stirred for $10 \mathrm{~min}$, following which a solution of tosylchloride $(35,0 \mathrm{~g}, 0.183$ $\mathrm{mol}$ ) in $25 \mathrm{~mL}$ of dichloroethane was added dropwise over a period of $20 \mathrm{~min}$. Thirty minutes after the completion of addition, the reaction was allowed to come to room temperature and left stirring overnight. The reaction was quenched by pouring onto $300 \mathrm{~mL}$ of distilled water. The organic layer was separated, and the aqueous layer was extracted with dichloromethane $(3 \times 50 \mathrm{~mL})$. The combined organic extracts were washed with distilled water to neutrality and dried over $\mathrm{Na}_{2} \mathrm{SO}_{4}$. Removal of the solvent in vacuo gave $3(25.1 \mathrm{~g}, 0.113 \mathrm{~mol}$; yield $76 \%)$ as colorless crystalline solid. Data in agreement with literature. ${ }^{2}$

m.p. 98-100 ${ }^{\circ} \mathrm{C} ;{ }^{1} \mathrm{H}$ NMR $\left(300 \mathrm{MHz}, \mathrm{CDCl}_{3}, 25^{\circ} \mathrm{C}\right): \delta=7.78-7.73(\mathrm{~m}, 2 \mathrm{H}, 1 / 2 \mathrm{AB}), 7.32-7.27(\mathrm{~m}$, $2 \mathrm{H}, 1 / 2 \mathrm{AB}$ ), 7.17 (t, $J=1.9 \mathrm{~Hz}, 2 \mathrm{H}, \mathrm{H}-2$ and H-5), 6.30 (t, $J=1.9 \mathrm{~Hz}, 2 \mathrm{H}, \mathrm{H}-3$ and H-4), 2.41 (s, $3 \mathrm{H}) ;{ }^{13} \mathrm{C} \mathrm{NMR}\left(\mathrm{CDCl}_{3}, 75 \mathrm{MHz}\right): \delta=145.4,136.6,130.4,127.2,121.1,113.9,22.0$; IR $\left(\mathrm{KBr}, \mathrm{cm}^{-}\right.$

\footnotetext{
${ }^{1}$ Allred, G. D.; Liebeskind, L. S.; Sanford, S. J. Am. Chem. Soc. 1996, 118, 2748-2749. Zhang, S.; Zhang, D.; Liebeskind, L. S. J. Org. Chem. 1997, 62, 2312-2313.

${ }^{2}$ Pleus, S.; Schwientek, M. Synth. Commun. 1997, 27, 2917-2930.
} 
$\left.{ }^{1}\right): 3146,1595,1454,1362,1169,1059,755 ; \mathrm{MS}(\mathrm{EI}+): \mathrm{m} / \mathrm{z}(\%): 221(95)\left[\mathrm{M}^{+}\right], 155(97)$ $\left[\mathrm{C}_{7} \mathrm{H}_{7} \mathrm{O}_{2} \mathrm{~S}^{+}\right], 91(100)\left[\mathrm{C}_{7} \mathrm{H}_{7}^{+}\right]$. Calcd for $\mathrm{C}_{11} \mathrm{H}_{11} \mathrm{NO}_{2} \mathrm{~S}$ : C 59.71, H 5.01, N 6.33. Found: C 59.68, $\mathrm{H}$ $5.02, \mathrm{~N} 6.31$.

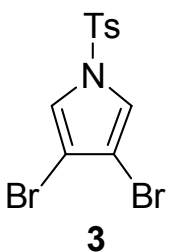

3,4-Dibromo-1-(toluene-4-sulfonyl)-1 $\boldsymbol{H}$-pyrrole (3). A solution of bromine (7.4 mL, $0.14 \mathrm{~mol}$ ) in acetic acid $(80 \mathrm{~mL})$ was slowly added to a solution of $2(13.5 \mathrm{~g}, 0.06 \mathrm{~mol})$ in acetic acid $(200 \mathrm{~mL})$ over $30 \mathrm{~min}$. After addition was completed the mixture was heated to reflux for 1.5 hours. The reaction mixture was warmed to room-temperature and the solvent removed under reduced pressure. The black oil was filtered through a column of silica using DCM as eluant. The resulting oil was recrystallised in a DCM/ether mixture giving $3(6.5 \mathrm{~g})$ as colorless crystals. The remaining oil was then purified using silica flash chromatography (DCM) affording further $3.5 \mathrm{~g}$ of product with an overall yield of $43 \%$.

m.p. $134-136{ }^{\circ} \mathrm{C} ;{ }^{1} \mathrm{H}$ NMR $\left(300 \mathrm{MHz}, \mathrm{CDCl}_{3}, 25^{\circ} \mathrm{C}\right): \delta=7.80-7.75(\mathrm{~m}, 2 \mathrm{H}, 1 / 2 \mathrm{AB}), 7.37-7.32(\mathrm{~m}$, $2 \mathrm{H}, 1 / 2 \mathrm{AB}), 7.19$ (s, $2 \mathrm{H}, \mathrm{H}-2$ and H-5), 2.44 (s, $3 \mathrm{H}) ;{ }^{13} \mathrm{C} \mathrm{NMR}\left(\mathrm{CDCl}_{3}, 75 \mathrm{MHz}\right): \delta=146.4$, 135.9, 130.8, 127.6, 120.4, 105.6, 22.1; IR (KBr, cm $\left.{ }^{-1}\right)$ : 3137, 1594, 1520, 1455, 1376, 1172, 1057 , 782. MS (EI+): $\mathrm{m} / \mathrm{z}(\%): 379(7)\left[\mathrm{M}^{+}\right], 155(74)\left[\mathrm{C}_{7} \mathrm{H}_{7} \mathrm{O}_{2} \mathrm{~S}^{+}\right], 91$ (100) $\left[\mathrm{C}_{7} \mathrm{H}_{7}^{+}\right]$. Calcd for $\mathrm{C}_{11} \mathrm{H}_{9} \mathrm{Br}_{2} \mathrm{NO}_{2} \mathrm{~S}: \mathrm{C} 34.85, \mathrm{H} 2.39, \mathrm{~N}$ 3.70. Found: C 34.86, H 2.38, N 3.73.

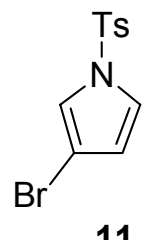

3-bromo-1-(toluene-4-sulfonyl)-1H-pyrrole (11). A solution of bromine $(0.1 \mathrm{~mL}, 2.1 \mathrm{mmol})$ in acetic acid $(5 \mathrm{~mL})$ was slowly added to a solution of $2(0.5 \mathrm{~g}, 2 \mathrm{mmol})$ in acetic acid $(10 \mathrm{~mL})$ over 30 min. After addition was completed the mixture was heated to reflux for 1 hours. The reaction mixture was warmed to room-temperature and the solvent removed under reduced pressure. The remaining oil was then purified using silica flash chromatography (DCM) affording 11 (0.440 g, $1.5 \mathrm{mmol}, 73 \%$ yield) as a colorless oil.

${ }^{1} \mathrm{H} \mathrm{NMR}\left(400 \mathrm{MHz}, \mathrm{CDCl}_{3}, 25^{\circ} \mathrm{C}\right): \delta=7.74-7.70(\mathrm{~m}, 2 \mathrm{H}, 1 / 2 \mathrm{AB}), 7.32-7.29(\mathrm{~m}, 2 \mathrm{H}, 1 / 2 \mathrm{AB}), 7.12$ $(\mathrm{dd}, J=1.5$ and $2.5 \mathrm{~Hz}, \mathrm{H}), 6.26(\mathrm{dd}, J=2.5$ and $3.3 \mathrm{~Hz}, \mathrm{H}), 7.12(\mathrm{dd}, J=1.5$ and $3.3 \mathrm{~Hz}, \mathrm{H}), 2.41$ $(\mathrm{s}, 3 \mathrm{H}) ;{ }^{13} \mathrm{C} \mathrm{NMR}\left(\mathrm{CDCl}_{3}, 75 \mathrm{MHz}\right): \delta=145.9,135.9,130.5,127.5,121.5,120.0,116.5,102.4$, 21.3; MS (EI+): m/z (\%): 301 (18) $\left[\mathrm{M}^{+}\right], 155$ (69) $\left[\mathrm{C}_{7} \mathrm{H}_{7} \mathrm{O}_{2} \mathrm{~S}^{+}\right], 91$ (100) $\left[\mathrm{C}_{7} \mathrm{H}_{7}{ }^{+}\right]$. Calcd for $\mathrm{C}_{11} \mathrm{H}_{10} \mathrm{BrNO}_{2} \mathrm{~S}: \mathrm{C} 44.01, \mathrm{H} 3.36, \mathrm{~N}$ 4.67. Found: C 39.38, H 3.33, N 4.69. 


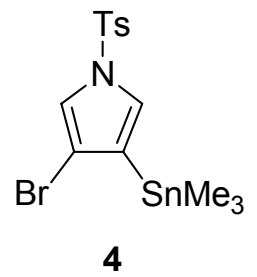

3-Bromo-1-(toluene-4-sulfonyl)-4-trimethylstanyl-1H-pyrrole (4). To a solution of 3 (5.2 g, $17.4 \mathrm{mmol})$ in dry THF $(170 \mathrm{~mL})$ at $-78{ }^{\circ} \mathrm{C}$ under an argon atmosphere was added a solution $1.7 \mathrm{M}$ in pentane $t$ - $\mathrm{BuLi}(2.0 \mathrm{~mL})$. After $30 \mathrm{~min}, \mathrm{Me}_{3} \mathrm{SnCl}(7.0 \mathrm{~g}, 34.8 \mathrm{mmol})$ was added. After 1 hour, the mixture was warmed to room temperature and the solvent was removed at reduced pressure. The crude product was chromatographed on silica gel (DCM/hexane 70/30) to afford 4 (6.4 g, 13.8 mmol, yield 79\%) as a colorless solid.

m.p. 88-90 ${ }^{\circ} \mathrm{C} ;{ }^{1} \mathrm{H}$ NMR $\left(300 \mathrm{MHz}, \mathrm{CDCl}_{3}, 25{ }^{\circ} \mathrm{C}\right): \delta=7.80-7.76(\mathrm{~m}, 2 \mathrm{H}, 1 / 2 \mathrm{AB}), 7.36-7.30(\mathrm{~m}, 2$ $\mathrm{H}, 1 / 2 \mathrm{AB}$ ), $7.24(\mathrm{~d}, J=2.2 \mathrm{~Hz}, 1 \mathrm{H}, \mathrm{H}-2), 6.94$ (d with side bands, $J=2.2 \mathrm{~Hz}, \mathrm{~J}_{\mathrm{H}, \mathrm{Sn}}=7.5 \mathrm{~Hz}, 1 \mathrm{H}$, $\mathrm{H}-5), 2.44$ (s, $3 \mathrm{H}), 0.32$ (s with side bands, $9 \mathrm{H}) ;{ }^{13} \mathrm{C} \mathrm{NMR}\left(\mathrm{CDCl}_{3}, 75 \mathrm{MHz}\right): \delta=145.7,136.1$, 130.5, 127.5, 126.3, 120.5, 113.9, 110.5, 22.1, -8.5; IR (KBr, $\left.\mathrm{cm}^{-1}\right): 3141,1595,1520,1473,1372$, 1173, 1055, 781. MS (EI+): m/z (\%): 463 (3) $\left[\mathrm{M}^{+}\right], 448$ (70) $\left[\mathrm{M}^{+}-\mathrm{CH}_{3}\right], 91$ (100) $\left[\mathrm{C}_{7} \mathrm{H}_{7}{ }^{+}\right]$. Calcd for $\mathrm{C}_{14} \mathrm{H}_{18} \mathrm{BrNO}_{2} \mathrm{SSn}$ : C 36.32, H 3.92, N 3.03. Found: C 36.30, H 3.95, N 3.00.

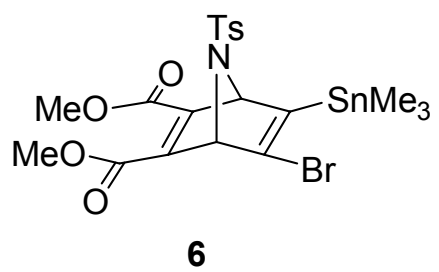

5-Bromo-7-(toluene-4-sulfonyl)-6-trimethylstannanyl-7-azabicyclo[2.2.1] hepta-2,5-diene-2,3dicarboxylic acid dimethyl ester (6). The bromo-tin pyrrole $4(970 \mathrm{mg}, 2.1 \mathrm{mmol})$ and dimethylacetylenedicarboxylate $(5 \mathrm{~mL})$ were combined in a sealed tube and heated at $120{ }^{\circ} \mathrm{C}$ for 12 hours. Excess of dimethylacetylenedicarboxylate was removed under vacuum and the crude mixture was separated by silica column chromatography (30\% ethyl acetate/hexanes) to yield the title compound 6 (840 mg, $1.8 \mathrm{mmol} ; 86 \%$ yield) as a colorless oil.

${ }^{1} \mathrm{H}$ NMR $\left(300 \mathrm{MHz}, \mathrm{CDCl}_{3}, 25{ }^{\circ} \mathrm{C}\right): \delta=7.61-7.56(\mathrm{~m}, 2 \mathrm{H}, 1 / 2 \mathrm{AB}), 7.31-7.26(\mathrm{~m}, 2 \mathrm{H}, 1 / 2 \mathrm{AB}), 5.46$ $(\mathrm{d}, 1 \mathrm{H}, J=2.4 \mathrm{~Hz}), 5.34(\mathrm{~d}, 1 \mathrm{H}, J=2.4 \mathrm{~Hz}), 3.79(\mathrm{~s}, 3 \mathrm{H}) 3.77(\mathrm{~s}, 3 \mathrm{H}), 2.43(\mathrm{~s}, 3 \mathrm{H}) ;{ }^{13} \mathrm{C} \mathrm{NMR}$ $\left(\mathrm{CDCl}_{3}, 75 \mathrm{MHz}\right): \delta=162.8,161.9,152.8,152.6,149.7,146.1,144.7,135.5,130.4,128.8,76.8$, 76.4, 52.9, 52.8, 22.0, -8.7; IR ( $\left.\mathrm{KBr} \mathrm{cm}^{-1}\right)$ : 3013, 2958, 1728, 1633, 1437, 1269, 1154, 1041, 894, 748, 678, 617; MS (EI+): m/z (\%): 463 (3) $\left[\mathrm{M}^{+}\right], 448$ (70) $\left[\mathrm{M}^{+}-\mathrm{CH}_{3}\right], 91(100)\left[\mathrm{C}_{7} \mathrm{H}_{7}^{+}\right]$. Calcd for $\mathrm{C}_{20} \mathrm{H}_{24} \mathrm{BrNO}_{6} \mathrm{SSn}: \mathrm{C} 39.70, \mathrm{H} 4.00, \mathrm{~N} 2.31$. Found: C 39.71, H 3.97 N 2.32.

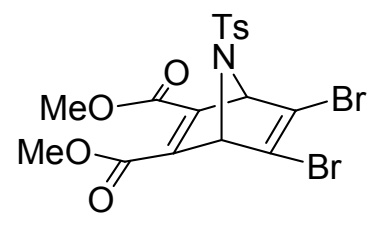

5

5,6-Dibromo-7-(toluene-4-sulfonyl)-7-azabicyclo[2.2.1] hepta-2,5-diene-2,3-dicarboxylic acid dimethyl ester (5). The dibromo pyrrole $3 \quad(500 \mathrm{mg}, 1.3 \mathrm{mmol})$ and dimethylacetylenedicarboxylate $(5 \mathrm{~mL})$ were combined in a sealed tube and heated at $150{ }^{\circ} \mathrm{C}$ for 12 
hours. Excess of dimethylacetylenedicarboxylate was removed under vacuum and the crude mixture was separated by silica column chromatography (20\% ethyl acetate/hexanes ) to yield the title compound as a yellow solid (450 mg, $0.9 \mathrm{mmol}$; yield $66 \%$ ).

m.p. $125-129^{\circ} \mathrm{C} ;{ }^{1} \mathrm{H}$ NMR $\left(300 \mathrm{MHz}, \mathrm{CDCl}_{3}, 25^{\circ} \mathrm{C}\right): \delta=7.67-7.62(\mathrm{~m}, 2 \mathrm{H}, 1 / 2 \mathrm{AB}), 7.35-7.30(\mathrm{~m}$, $2 \mathrm{H}, 1 / 2 \mathrm{AB}), 5.37(\mathrm{~s}, 2 \mathrm{H}), 3.84(\mathrm{~s}, 6 \mathrm{H}), 2.44(\mathrm{~s}, 3 \mathrm{H}) ;{ }^{13} \mathrm{C} \mathrm{NMR}\left(\mathrm{CDCl}_{3}, 75 \mathrm{MHz}\right): \delta=161.7,150.9$, 145.1, 133.6, 130.7, 128.9, 133.0, 76.5, 53.2, 22.0; IR ( $\left.\mathrm{KBr} \mathrm{cm}^{-1}\right)$ : 3023, 2950, 1709, 1631, 1439,

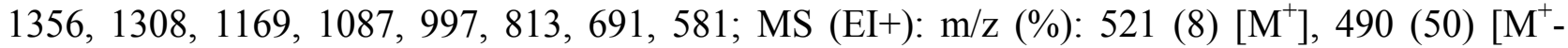
$\left.\mathrm{OCH}_{3}\right], 155$ (100) $\left[\mathrm{C}_{7} \mathrm{H}_{7} \mathrm{O}_{2} \mathrm{~S}^{+}\right]$. Calcd for $\mathrm{C}_{17} \mathrm{H}_{15} \mathrm{Br}_{2} \mathrm{NO}_{6} \mathrm{~S}$ : C 39.18, H 2.90, N 2.69. Found: C $39.17, \mathrm{H} 2.88$ N 2.72.

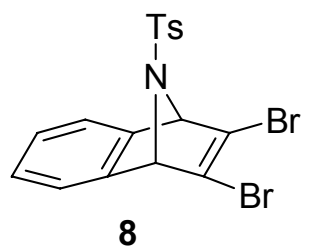

2,3-Dibromo-9-(toluene-4-sulfonyl)-1,4-dihydro-1,4-epiazano-naphthalene (8). In a 3 necked round bottom flask with a reflux condenser and dropping tunnel, under Ar atmosphere to a solution of isoamylnitrite $(6.3 \mathrm{~mL}, 47 \mathrm{mmol})$ in DCM $(50 \mathrm{ml})$ was added dropwise a solution of anthranilic acid $(4.3 \mathrm{~g}, 31 \mathrm{mmol})$ and $3(4.0 \mathrm{~g}, 10.5 \mathrm{mmol})$ in acetone $(80 \mathrm{ml})$. The addition took about 30 minutes. The reaction was allowed to stir at $50{ }^{\circ} \mathrm{C}$ overnight. The solvent was removed in vacuo and the mixture was dissolved in DCM. The solution was washed with water $(3 \times 50 \mathrm{~mL})$, brine and dried over $\mathrm{MgSO}_{4}$ and concentrated. The black oil was purified by column chromatography (DCM/hexane 7:3) affording 8 (2.0 g, $4.3 \mathrm{mmol}, 43 \%$ yield)as a yellow solid.

m.p. $200-203{ }^{\circ} \mathrm{C} ;{ }^{1} \mathrm{H}$ NMR $\left(300 \mathrm{MHz}, \mathrm{CDCl}_{3}, 25{ }^{\circ} \mathrm{C}\right): \delta=7.64-7.60(\mathrm{~m}, 2 \mathrm{H}, 1 / 2 \mathrm{AB}), 7.30-7.22(\mathrm{~m}$, $4 \mathrm{H}), 7.02-6.99(\mathrm{~m}, 2 \mathrm{H}, 1 / 2 \mathrm{AB}), 5.38$ (s, $2 \mathrm{H}), 2.42(\mathrm{~s}, 3 \mathrm{H}) ;{ }^{13} \mathrm{C} \mathrm{NMR}\left(\mathrm{CDCl}_{3}, 75 \mathrm{MHz}\right): \delta=$ 144.9, 144.4, 135.4, 133.6, 130.4, 128.8, 126.6, 121.5, 74.4, 22.0; IR (KBr): 3275, 3081, 2920,

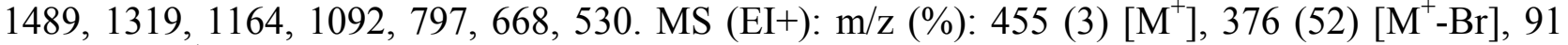
(100) $\left[\mathrm{C}_{7} \mathrm{H}_{7}^{+}\right]$. Calcd for $\mathrm{C}_{17} \mathrm{H}_{13} \mathrm{Br}_{2} \mathrm{NO}_{2} \mathrm{~S}$ : C 44.86, H 2.88, N 3.08. Found: C 44.85, H 2.90 N 3.06.

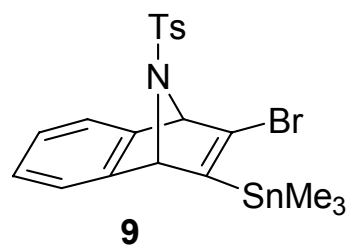

\section{2-Bromo-9-(toluene-4-sulfonyl)-3-trimethylstannanyl-1,4-dihydro-1,4-epiazanonaphthalene}

(9). A $2.5 \mathrm{M}$ solution of $n$-BuLi in hexane $(2.6 \mathrm{ml}, 6.5 \mathrm{mmol})$ was added dropwise to a solution of $9(2.2 \mathrm{~g}, 5 \mathrm{mmol})$ in $40 \mathrm{~mL}$ of dry THF at $-78^{\circ} \mathrm{C}$ under Ar atmosphere. The mixture was stirred for 30 minutes and $\mathrm{Me}_{3} \mathrm{SnCl}$ was added portionwise and the mixture stirred overnight. The crude product was concentrated in vacuo. The product was purified with flash chromatography using DCM as eluant giving 9 (2.1 g, $3.9 \mathrm{mmol}, 77 \%$ yield $)$ as a colorless oil.

${ }^{1} \mathrm{H}$ NMR $\left(300 \mathrm{MHz}, \mathrm{CDCl}_{3}, 25^{\circ} \mathrm{C}\right): \delta=7.57-7.52(\mathrm{~m}, 2 \mathrm{H}, 1 / 2 \mathrm{AB}), 7.21-7.15(\mathrm{~m}, 3 \mathrm{H}, 1 / 2 \mathrm{AB}+$ $\left.\mathrm{H}_{\mathrm{ar}}\right)$, 7.05-7.00 (m, $\left.1 \mathrm{H}, \mathrm{H}_{\mathrm{ar}}\right), 6.90-6.85\left(\mathrm{~m}, 2 \mathrm{H}, \mathrm{H}_{\mathrm{ar}}\right), 5.55(\mathrm{~d}, J=1.1 \mathrm{~Hz}, 1 \mathrm{H}, \mathrm{H}-5), 5.26(\mathrm{~d}, J=$ $1.1 \mathrm{~Hz}, 1 \mathrm{H}), 2.39(\mathrm{~s}, 3 \mathrm{H}) ;{ }^{13} \mathrm{C} \mathrm{NMR}\left(\mathrm{CDCl}_{3}, 75 \mathrm{MHz}\right): \delta=153.1,146.7,146.2,145.5,143.9,136$. $130.0,128.7,126.1,125.4,121.7,120.5,74.7,74.4,21.9,-8.8$; IR (KBr): 2922, 1492, 1346, 1163 , 


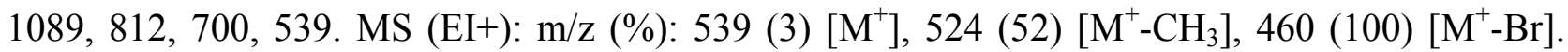
Calcd for $\mathrm{C}_{20} \mathrm{H}_{22} \mathrm{BrNO}_{2} \mathrm{SSn}$ : C 44.56, H 4.11, N 2.60. Found: C 44.57, H 4.13 N 2.59.

General procedure for the cyclotrimerisation of stannylbromobicyclic systems. To a solution of the stannylbromo compound $(3.3 \mathrm{mmol})$ in dry NMP $(18 \mathrm{~mL})$, maintained at $-20{ }^{\circ} \mathrm{C}$ under $\mathrm{Ar}, \mathrm{CuTC}$ $(0.87 \mathrm{~g}, 5.0 \mathrm{mmol})$ was added portionwise. The well-stirred mixture was left to rise to rt overnight. The resulting slurry was diluted with $20 \%$ aqueous $\mathrm{NH}_{3}(50 \mathrm{~mL})$ and extracted with AcOEt $(3 \times 50$ $\mathrm{mL})$. Combined organic extracts were washed with $\mathrm{H}_{2} \mathrm{O}(50 \mathrm{~mL})$, brine $(50 \mathrm{~mL})$, dried over $\mathrm{MgSO}_{4}$ and concentrated in vacuum to afford an oil that was purified by flash-chromatography.

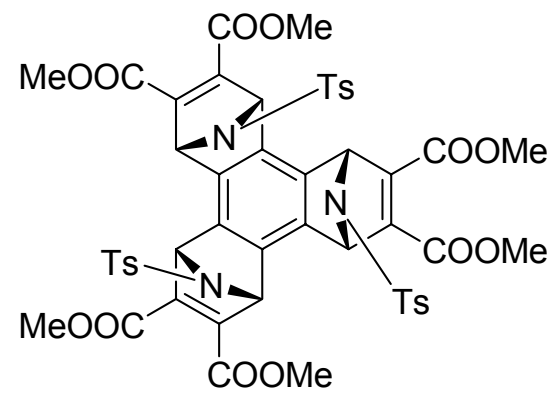

syn-7

Syn-7. Eluant AcOEt:DCM (5:95), (37\% yield) as orange crystals. m.p. $189{ }^{\circ} \mathrm{C}$ (dec.); ${ }^{1} \mathrm{H}$ NMR $\left(300 \mathrm{MHz}, \mathrm{CDCl}_{3}, 25^{\circ} \mathrm{C}\right): \delta=7.59-7.54(\mathrm{~m}, 6 \mathrm{H}, 1 / 2 \mathrm{AB}), 7.29-7.24(\mathrm{~m}, 6 \mathrm{H}, 1 / 2 \mathrm{AB}), 5.76(\mathrm{~s}, 6 \mathrm{H})$, $3.65(\mathrm{~s}, 18 \mathrm{H}), 2.40(\mathrm{~s}, 9 \mathrm{H}) ;{ }^{13} \mathrm{C} \mathrm{NMR}\left(\mathrm{CDCl}_{3}, 75 \mathrm{MHz}\right): \delta=161.6,147.1,145.1,134.3,133.6$, 130.6, 128.3, 68.1, 52.7, 21.9; IR $\left(\mathrm{KBr} \mathrm{cm}^{-1}\right): 3035,2953,1734,1632,1597,1436,1352,1296$, 1170, 1089, 992, 815, 706, 663, 575. Calcd for $\mathrm{C}_{51} \mathrm{H}_{45} \mathrm{~N}_{3} \mathrm{O}_{18} \mathrm{~S}_{3}$ : C 56.50, H 4.18, N 3.88. Found: C $56.53, \mathrm{H} 4.20 \mathrm{~N} 3.85$.

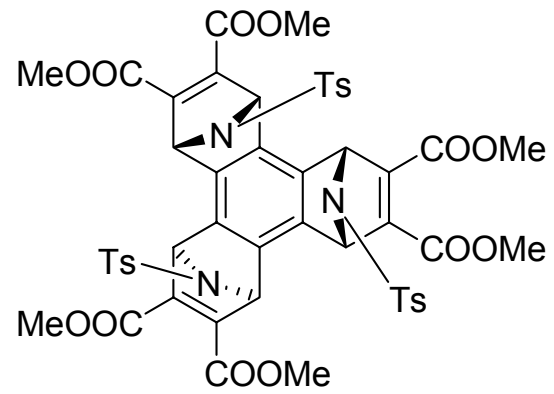

anti-7

Anti-7. Eluant AcOEt:DCM (5:95), (44\% yield) as white solid. m.p. $201{ }^{\circ} \mathrm{C}$ (dec.) ${ }^{1} \mathrm{H}$ NMR $(300$ $\left.\mathrm{MHz}, \mathrm{CDCl}_{3}, 25^{\circ} \mathrm{C}\right): \delta=7.56-7.46(\mathrm{~m}, 2 \mathrm{H}, 1 / 2 \mathrm{AB}), 7.42-7.37(\mathrm{~m}, 4 \mathrm{H}, 1 / 2 \mathrm{AB}), 7.17-7.12(\mathrm{~m}, 6 \mathrm{H}$, $21 / 2 \mathrm{AB}), 5.72(\mathrm{~s}, 2 \mathrm{H}), 5.66(\mathrm{~s}, 4 \mathrm{H}), 3.96(\mathrm{~s}, 6 \mathrm{H}), 3.76(\mathrm{~s}, 6 \mathrm{H}), 3.74(\mathrm{~s}, 6 \mathrm{H}), 2.34(\mathrm{~s}, 3 \mathrm{H}), 2.34(\mathrm{~s}$, $6 \mathrm{H}) ;{ }^{13} \mathrm{C} \mathrm{NMR}\left(\mathrm{CDCl}_{3}, 75 \mathrm{MHz}\right): \delta=161.7,161.6,161.4,147.9,147.6,145.3,145.0,134.3$, 134.2, 134.0, 133.5, 133.4, 130.6, 130.3, 128.3, 128.0, 68.3, 68.1, 67.8, 53.3, 53.0, 52.9, 21.9 (2 carbons); IR (KBr cm $\left.{ }^{-1}\right): 2955,2925,1740,1717,1631,1597,1437,1353,1297,1262,1164,1089$, 815, 706, 666, 575. Calcd for $\mathrm{C}_{51} \mathrm{H}_{45} \mathrm{~N}_{3} \mathrm{O}_{18} \mathrm{~S}_{3}$ : C 56.50, H 4.18, N 3.88. Found: C 56.52, H $4.21 \mathrm{~N}$ 4.01 . 


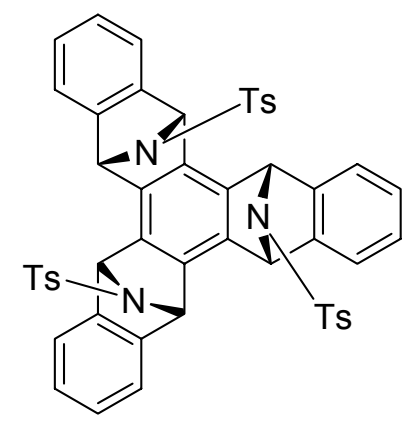

syn-10

syn-10. Eluant AcOEt:Hexane (1:1), (19\% yield) as red crystals. m.p. $200{ }^{\circ} \mathrm{C}(\mathrm{dec} .)^{1} \mathrm{H}$ NMR $(300$ $\left.\mathrm{MHz}, \mathrm{CDCl}_{3}, 2{ }^{\circ} \mathrm{C}\right): \delta=7.45-7.44(\mathrm{~m}, 6 \mathrm{H}, 1 / 2 \mathrm{AB}), 7.12-7.08(\mathrm{~m}, 6 \mathrm{H}, 1 / 2 \mathrm{AB}), 6.82-6.79(\mathrm{~m}, 6 \mathrm{H}$, $1 / 2$ AA'BB'), 6.63-6.59 (m, $6 \mathrm{H}, 1 / 2$ AA'BB'), 5.79 (s, $6 \mathrm{H}), 2.32(\mathrm{~s}, 9 \mathrm{H}) ;{ }^{13} \mathrm{C} \mathrm{NMR}^{\prime}\left(\mathrm{CDCl}_{3}, 75\right.$ $\mathrm{MHz}): \delta=144.8,144.1,134.5,134.1,130.0,128.2,126.7,121.1,66.4,21.9$; IR $\left(\mathrm{KBr} \mathrm{cm}^{-1}\right): 3053$, 2923, 1734, 1339, 1251, 1158, 1089, 991, 814, 689, 577. Calcd for $\mathrm{C}_{51} \mathrm{H}_{39} \mathrm{~N}_{3} \mathrm{O}_{6} \mathrm{~S}_{3}$ : C 69.13, H 4.44, N 4.74. Found: C 69.11, H 4.31 N 4.75.

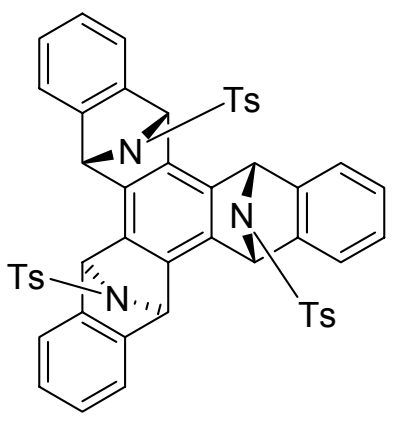

anti-10

anti-10. Eluant AcOEt:Hexane (1:1), (57\% yield) as colorless crystals. m.p. $205{ }^{\circ} \mathrm{C}(\mathrm{dec} .)^{1} \mathrm{H}$ NMR $\left(300 \mathrm{MHz}, \mathrm{CD}_{3} \mathrm{COCD}_{3}, 25^{\circ} \mathrm{C}\right): \delta=7.60-7.56\left(\mathrm{~m}, 2 \mathrm{H}, 1 / 2 \mathrm{AA}{ }^{\prime} \mathrm{BB}^{\prime}\right), 7.35-7.31(\mathrm{~m}, 2 \mathrm{H}, 1 / 2 \mathrm{AB})$, 7.29-7.25 (m, 2 H, 1/2 AA'BB'), 7.13-7.08 (m, 8 H, 1/2 AB), 6.67-6.63 (m, 8 H, 1/2 AB), 6.20 (s, 2 H), $6.12(\mathrm{~s}, 2 \mathrm{H}), 6.05(\mathrm{~s}, 2 \mathrm{H}), 5.95-5.91(\mathrm{~m}, 2 \mathrm{H}, 1 / 2 \mathrm{AB}), 2.87(\mathrm{~s}, 3 \mathrm{H}), 2.83(\mathrm{~s}, 6 \mathrm{H}) ;{ }^{13} \mathrm{C} \mathrm{NMR}\left(\mathrm{CDCl}_{3}\right.$, $75 \mathrm{MHz}): \delta=146.8,146.3,146.0,143.4,142.4,135.1,135.0,134.6,134.5,134.3,129.7,129.2$, 127.7, 127.2, 126.8, 126.6, 126.4, 122.0, 121.9, 121.5, 66.6, 66.3, 66.0, 20.8, 20.7; IR ( $\left.\mathrm{KBr} \mathrm{cm}^{-1}\right)$ : 3043, 2955, 2925, 1740, 1717, 1353, 1262, 1164, 1089, 1024, 706, 575, 542. Calcd for $\mathrm{C}_{51} \mathrm{H}_{39} \mathrm{~N}_{3} \mathrm{O}_{6} \mathrm{~S}_{3}$ : C 69.13, H 4.44, N 4.74. Found: C 69.12, H 4.41 N 4.76. 

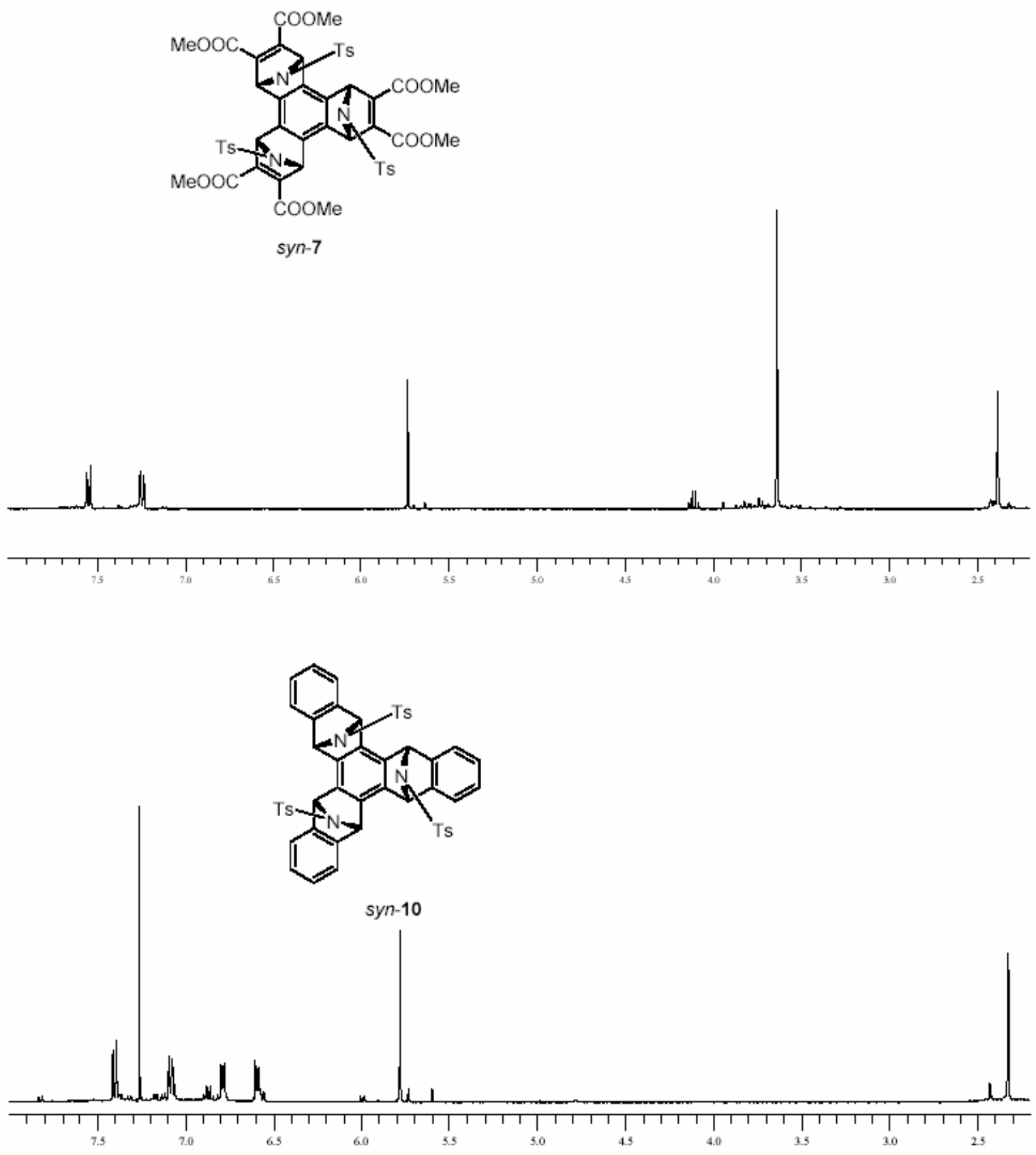